\title{
Rapid Methods for the Detection of Bacteria via Giant Magnetoresistance Sensor and Biomagnetic Marker
}

Hamidreza Shirzadfar*

Department of Biomedical Engineering, Sepahan University, Isfahan, Iran

In recent years, research and development of new sensors have experienced very strong growth in some scientific disciplines, particularly in the biomedical field.

The giant magnetoresistance effect has been described independently in 1988 by Professors Albert Fert and Professor Peter Grunberg and they received the Nobel Prize for Physics in 2007 for their work in this area.

The nature of the magnetoresistive effect is a significant change in resistance (10-20\%) of multilayer structures of magnetic and nonmagnetic metals, under the effect of an external magnetic field. The basis for this phenomenon is due to the property of quantum electron intrinsic angular momentum or spin [1].

The GMR phenomenon of the basic effect is to significantly reduce the resistance of the alternating in the multilayer structure of the sample layers, when they are exposed to an external magnetic field.

Since its description in 1988 , due to their small size, low power consumption, robustness in harsh conditions and relatively low cost, many sensors are developed or designed on the basis of the GMR effect are increasingly used in industrial electronics, IT, automotive industry, as well as equipment medical diagnosis. Additionally, GMR sensors have a high sensitivity and detection capability for measuring weak fields.

The development of components for nanoscale manufacturing methods have created devices successfully using this property of the electron. This was the beginning of a new scientific field; spintronics. The emergence of sensors based on the GMR effect has especially enabled a strong increase in the recording density of computer hard disks. But nowadays, it is no longer the sole domain of application.

The GMR effect is generated by the quantum properties of electrons, which have characteristics such as electron spin. Electrons can have only two possible directions: up or down. The conduction electrons of spin whose direction of spin is the same as the direction of the magnetic field inside the GMR encounter less resistance to their motion and have more freedom of movement than the electrons with spin oriented against the internal magnetic fields, which encounter greater resistance to their motion, and often collide with atoms in their environment. In the first case, the electrical resistance of the environment will be less than in the second one. The magnetoresistance is observed when an electric current flows in a ferromagnetic material at low temperatures. This can be explained by the fact that, under certain conditions, there is a strong difference between the resistivity $\rho \uparrow$ for electrons whose spins are parallel to the direction of magnetization and resistivity $\rho \downarrow$ for electrons with spins pointing in the antiparallel direction.

The giant magnetoresistance effect is observed in multilayer materials with alternating thin layers of ferromagnetic and nonmagnetic metals.

The thickness of a single ultra-thin layer may be only a few nanometers. The resistance of a sample reaches its maximum when local magnetic fields in ferromagnetic materials are in antiparallel and a minimum when they are in parallel. This phenomenon was named giant magnetoresistance, as resistance value was much greater than known magnetoresistance.

Magnetic fluids are colloidal solutions of magnetic materials in minute sizes. Magnetic fluids are unique because they combine mobility and a higher magnetization than ordinary liquids. Each particle is coated with a thin protective layer that prevents the adhesion of other particles. These particles spread throughout the entire volume of the liquid can absorb a positive charge and produce repulsive electrostatic forces.

When a magnetic field is applied to a ferrofluid, the particles of the magnetic moments align with the field almost instantaneously. The magnetization of a magnetic fluid responds instantaneously to changes in the applied magnetic field, and when the latter is removed, the magnetic dipole of each particle reorients randomly.

Today, magnetic fluids are used in various technological applications such as the chemical industry (as lubricants in magnetic cooling systems), medicine (magnetic separation of cells, cancer treatment, magnetic drug delivery and immunoassay), in mechanical engineering (NASA research projects on the use of a ferromagnetic fluid in a closed ring, as the basis for a spacecraft stabilization system in space and in the printing industry and the industry oil), electronics, mining and military industry (sealing and insulation gaps between them in pieces movement, friction reduction) [2].

As mentioned before, the most important use of ferrofluids is in medicine. As we know, anti-cancer drugs are harmful to healthy cells. However, by mixing with a magnetic fluid before injecting them into the blood, it is possible inter to a tumor without damaging surrounding tissues. The magnetite particles are used to treat cancer by hyperthermia method. This method of treatment is based on the fact that, under the influence of a magnetic field, alternating magnetite particles causes their heating and inhibits growth of cancer cells [3].

A magnetic fluid can also be used as a contrast agent for X-ray fluoroscopy. The usual diagnostics of the gastrointestinal tract are based on the use of barium sulfate (baryta slurry). Another major application of ferrofluids is in surgery. A magnetic fluid can be injected into a vein or artery and then positioned using a magnet to produce a cap for

*Corresponding author: Hamidreza Shirzadfar, Department of Biomedical Engineering, Sepahan University, Isfaha, Iran, Tel: +983136530125; Fax: +983136530245; E-mail: h.shirzadfar@sepahan.ac.ir

Received May 25, 2016; Accepted May 26, 2016; Published May 29, 2016

Citation: Shirzadfar H (2016) Rapid Methods for the Detection of Bacteria via Giant Magnetoresistance Sensor and Biomagnetic Marker. J Bioanal Biomed 8: e141. doi:10.4172/1948-593X.1000e141

Copyright: @ 2016 Shirzadfar H. This is an open-access article distributed under the terms of the Creative Commons Attribution License, which permits unrestricted use, distribution, and reproduction in any medium, provided the original author and source are credited. 
Citation: Shirzadfar H (2016) Rapid Methods for the Detection of Bacteria via Giant Magnetoresistance Sensor and Biomagnetic Marker. J Bioanal Biomed 8: e141. doi:10.4172/1948-593X.1000e141

closing a vein after ablation. Another vital role of magnetic fluids, that its use in orthopaedics and traumatology where it is used to lubricate the joints (for lower limb prostheses) [4].

As a new application in medicine, it is recommended to use the GMR sensor and a specific smart bio magnetic marker to decrease the detection time of bacterial pathogens like Escherichia coli O157:H7. This method also permits us to improve accuracy detection of bacteria comparing other ordinary laboratory methods [5-7].

At the end, it should be noted that the proposed method by GMR sensors and magnetic marker increased the speed, accuracy and measurement comfort compared other similar equipment, as SQUID or VSM. This is explained because of the microscopic size of the sensor, the appropriate sensitivity and the ability to measure in a weak magnetic field [7].

This research permits to consider development of biosensors or measuring devices further magnetic properties of existing with essential features like the ability to work on a microscopic scale and broadband frequencies in magneto-spectroscopy.

\section{References}

1. Baibich MN, Broto JM, Fert A, Van Dau FN, Petroff F, et al. (1988) Giant Magnetoresistance of $(001) \mathrm{Fe} /(001) \mathrm{Cr}$ Magnetic Superlattices. Physical Review Letters 61: 2472-2475.

2. Scherer C, Neto AMF (2005) Ferrofluids: Properties and applications. Brazilian Journal of Physics 35: 718-727.

3. Hergt R, Andra W, D'Ambly CG, Hilger I, Kaiser WA, et al. (1998) Physicallimits of hyperthermia using magnetite fine particles. leee Transactions on Magnetics 34: $3745-3754$.

4. Yu W, Xie HQ (2012) A Review on Nanofluids: Preparation, Stability Mechanisms, and Applications. Journal of Nanomaterials.

5. Shirzadfar H (2014) Design and evaluation of a GMR-biosensor for magnetic characterization of biological medium. PhD thesis Université de Lorraine.

6. Shirzadfar H, Nadi M, Kourtiche D, Yamada S, Shahabi P (2015) Characterization Of A Needle-Type Giant Magnetoresistance Sensor For Detection Of Escherichia Coli's Magnetic Marker. International Journal On Smart Sensing And Intelligent Systems 8: 220-234.

7. Shirzadfar H, Nadi M, Kourtiche. D, Yamada S, Hauet T (2015) Needle-type GMR sensor to estimate the magnetic properties of diluted ferrofluid for biomedicine application. IRBM 36: 178-184. 